\title{
Posterior Reversible Encephalopathy Syndrome and brain haemorrhage as COVID-19 complication: a review of the available literature
}

\author{
Francesco Motolese ${ }^{1}$ (D) Mario Ferrante ${ }^{1} \cdot$ Mariagrazia Rossi $^{1} \cdot$ Alessandro Magliozzi $^{1} \cdot$ Martina Sbarra $^{2}$. \\ Francesca Ursini ${ }^{1}$. Massimo Marano ${ }^{1}$ - Fioravante Capone ${ }^{1}$. Francesco Travaglino ${ }^{3}$. Raffaele Antonelli Incalzi ${ }^{4}$. \\ Vincenzo Di Lazzaro' ${ }^{1}$ Fabio Pilato ${ }^{1}$
}

Received: 20 March 2021 / Revised: 11 July 2021 / Accepted: 12 July 2021 / Published online: 21 July 2021

(c) Springer-Verlag GmbH Germany, part of Springer Nature 2021

\begin{abstract}
Background SARS-CoV-2 infection has been associated with various neurological manifestations. Since patients affected by SARS-CoV-2 infection present coagulation and immune system dysregulation, ischemic or haemorragic stroke is not uncommon, irrespective of respiratory distress. However, the occurrence of focal neurological deficits together with other symptoms like headache, cortical blindness, seizure and altered mental status should prompt the diagnosis of Posterior Reversible Encephalopathy Syndrome (PRES). Antithrombotic treatment, the alteration of endothelial function, and coagulopathy due to COVID-19 and PRES leading to the breakdown of blood-brain barrier may then contribute to the occurrence of a brain haemorrhage.

Methods We describe the case of a COVID-19 patient who developed bilateral occipital lobe haemorrhages suggestive of haemorrhagic PRES. We then reviewed the available literature about haemorrhagic evolution of PRES in COVID-19.

Results We describe the clinical and radiological features of five COVID-19 patients who developed haemorrhagic PRES. Conclusions Coagulopathy and endothelial dysfunction resulting from the massive release of cytokines during the host immune response may be key factors in the pathogenesis of COVID-19-related PRES. Antithrombotic therapy and the leakage of the blood-brain barrier can subsequently increase the risk of haemorrhagic transformation of the lesioned brain tissue. A prompt diagnosis of PRES is mandatory, since the timely interruption/reversal of antithrombotic therapy may be a key determinant for a good prognosis.
\end{abstract}

Keywords Posterior Reversible Encephalopathy Syndrome $\cdot$ PRES $\cdot$ Haemorrhage $\cdot$ COVID-19 $\cdot$ SARS-CoV-2

Francesco Motolese

f.motolese@unicampus.it

1 Neurology, Neurophysiology and Neurobiology Unit, Department of Medicine, Università Campus Bio-Medico di Roma, Viale Alvaro del Portillo, 21, 00128 Rome, Italy

2 Departmental Faculty of Medicine and Surgery, Unit of Diagnostic Imaging and Interventional Radiology, Università Campus Bio-Medico di Roma, Rome, Italy

3 Emergency Department, Università Campus Bio-Medico di Roma, Rome, Italy

4 Division of Geriatric Medicine, Università Campus Bio-Medico di Roma, Rome, Italy

\section{Introduction}

SARS-CoV-2 infection has been associated with various neurological manifestations. These include a wide spectrum of diseases such as encephalopathy, stroke, acute neuropathies (e.g. Guillain-Barrè syndrome), encephalitis or encephalomyelitis, seizures, and status epilepticus [1,2]. While it is still debated if neurological sequelae are due to direct infection of SARS-CoV-2 on the central nervous system (CNS) or if neurological involvement is mediated by activation of the immune system, cerebrovascular events are well-known complications of Coronavirus disease (COVID-19) [3-5]. Since patients affected by SARS-CoV-2 infection present coagulation and immune system dysregulation, stroke is not uncommon, regarding $0.9-2.7 \%$ of all COVID-19 cases, irrespective of respiratory distress. Spontaneous intracranial 
haemorrhage accounts for about 20\% of all COVID-19 strokes and is often associated with severe pneumonia [6].

The occurrence of focal neurological deficits during SARS-CoV-2 infection suggests acute stroke but if other symptoms like headache, cortical blindness, seizure and altered mental status are present, Posterior Reversible Encephalopathy Syndrome (PRES) should be also considered in the differential diagnosis. Herein, we present the case of a patient affected by COVID-19-related pneumonia who developed bilateral occipital lobe haemorrhages suggestive of haemorrhagic PRES.

\section{Case report}

A 74-year-old woman was admitted to the emergency department because of dyspnea, cough and fever. An RTPCR nasopharyngeal swab resulted positive for SARS-CoV2 infection and chest CT showed multifocal pneumonia. Medical history was remarkable for hypothyroidism, dyslipidemia and atrial fibrillation in treatment with Vitamin-K antagonist
(VKA). Supplemental oxygen was needed $-3 \mathrm{~L} / \mathrm{min}$ nasal cannula - and therapy with Methylprednisolone $60 \mathrm{mg} / \mathrm{die}$, Ceftriaxone and Clarithromycin was started. VKA was discontinued and replaced by enoxaparin 6000 UI twice a day.

On the fifth day of hospitalization the acute onset of confusion, disorientation and cortical blindness, with no other focal neurological deficits, occurred. All vital parameters were unremarkable, but blood pressure was $170 / 100 \mathrm{mmHg}$. Brain CT scan showed bilateral vasogenic edema and intraparenchymal hemorrhages in the occipital lobes (Fig. 1). CT angiography did not reveal any arterial and venous vessels malformations. Blood examinations were remarkable for leukocytosis, thrombocytosis and alteration of C-reactive protein and coagulation profile (Table 1). Anticoagulation reversal therapy with Phytomenadione $20 \mathrm{mg}$ was administered. Besides, Enoxaparin was stopped, and strict blood pressure control was implemented. Dexamethasone $8 \mathrm{mg}$ twice per day in place of Methylprednisolone was started, together with antiepileptic prophylaxis with Levetiracetam.

After few hours, there was a sudden deterioration of the state of consciousness, a brain CT scan was repeated showing

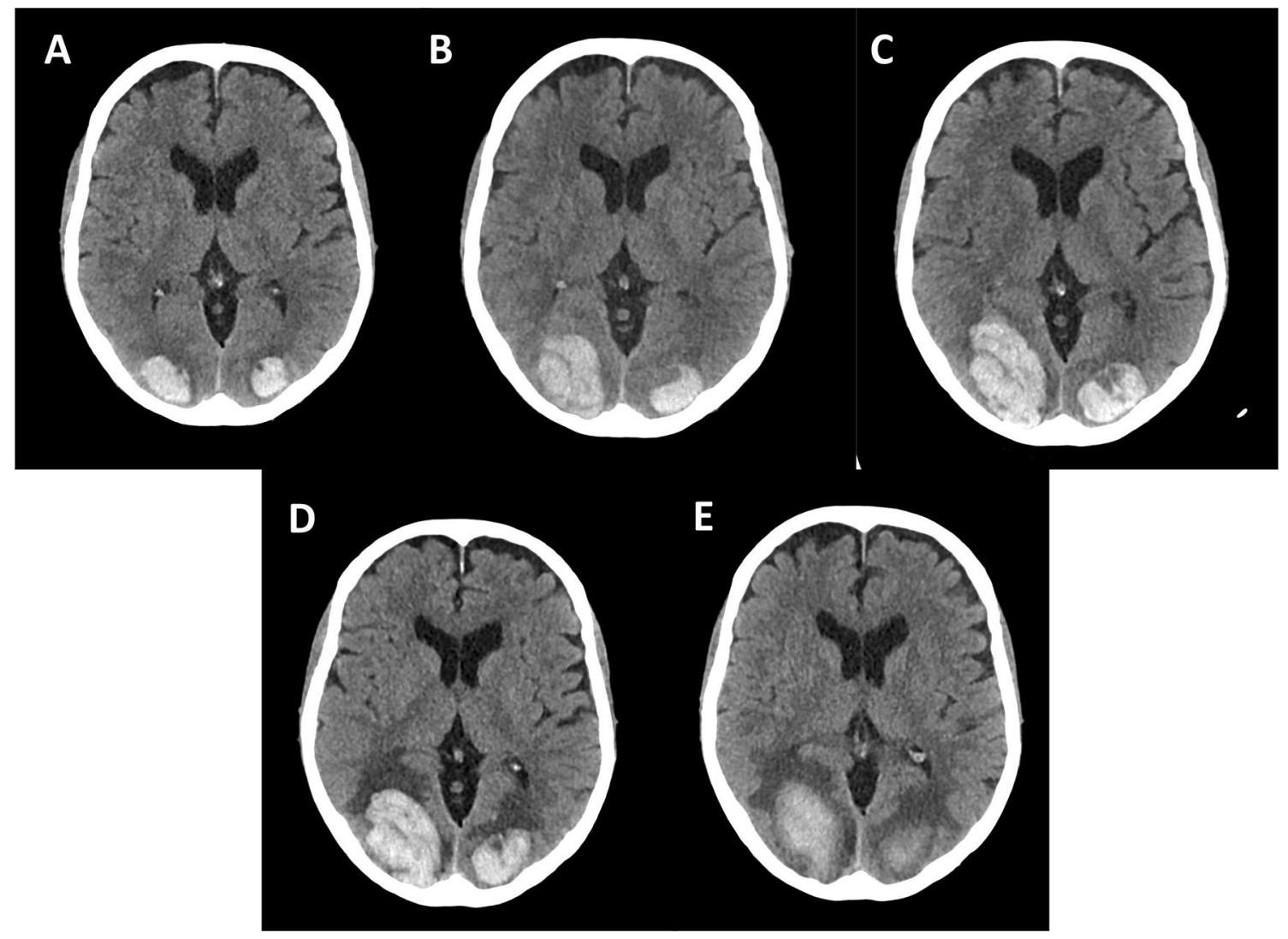

Fig. 1 Intraparenchymal hemorrhage with occipital pattern in our patient, at the onset (A), after $4 \mathrm{~h}(\mathbf{B})$, after 2 days (C), after 7 days (D), and after 20 days (E). Axial CT scan (A) demonstrates bilateral occipital lobes hematomas with surrounding vasogenic edema. Vascular malformations were not detected on CT angiography (not shown). After $4 \mathrm{~h}$ (B) and 2 days (C), axial CT scans show an increase of hematomas volume, especially in the right occipital lobe with a slight mass effect on the right lateral ventricle. After 7 days (D) and 20 days (E) axial CT scans show a progressive decrease in the density and size of hematomas, as for the physiological evolution 
Table 1 Laboratory data

\begin{tabular}{|c|c|c|}
\hline & $\begin{array}{l}\text { Reference range, adults, this } \\
\text { hospital }\end{array}$ & $\begin{array}{l}5 \text { Days from } \\
\text { hospitalization, this } \\
\text { hospital }\end{array}$ \\
\hline Hemoglobin (g/dl) & $12-16$ & 14,80 \\
\hline Hematocrit (\%) & $36-46$ & 46,30 \\
\hline White-cell count (per $\mu \mathrm{l})$ & $4000-10,000$ & 24,770 \\
\hline \multicolumn{3}{|l|}{ Differential count $($ per $\mu \mathrm{l})$} \\
\hline Neutrophils & $2000-7000$ & 22,010 \\
\hline Lymphocytes & $1000-3000$ & 1450 \\
\hline Monocytes & $200-1000$ & 1270 \\
\hline Eosinophils & $20-500$ & 0 \\
\hline Basophils & $20-100$ & 40 \\
\hline Platelet count (per $\mu \mathrm{l})$ & $150,000-400,000$ & 529,000 \\
\hline aPTT (s) & $23,00-32,00$ & 38,50 \\
\hline $\mathrm{PT}(\mathrm{s})$ & $9,00-14,00$ & 32,30 \\
\hline INR & $0,80-1,20$ & 2,56 \\
\hline Fibrinogen (mg/dl) & $200-400$ & 546 \\
\hline D-dimer (ng/ml) & $0-500$ & 440 \\
\hline Sodium $(\mathrm{mmol} / \mathrm{L})$ & $136-145$ & 139 \\
\hline Potassium (mmol/L) & $3,50-5,10$ & 3,90 \\
\hline Urea nitrogen $(\mathrm{mg} / \mathrm{dl})$ & $21,40-42,80$ & 33,10 \\
\hline Creatinine $(\mathrm{mg} / \mathrm{dl})$ & $0,55-1,02$ & 0,62 \\
\hline Glucose (mg/dl) & $82-115$ & 105 \\
\hline Alanine aminotransferase (U/L) & $0-55$ & 74 \\
\hline Aspartate aminotransferase (U/L) & $5-34$ & 94 \\
\hline Ferritin (ng/ml) & $4,63-204,00$ & 361,00 \\
\hline C-reactive protein $(\mathrm{mg} / \mathrm{dl})$ & $<0,50$ & 0,78 \\
\hline Procalcitonin (ng/ml) & $<0,50$ & 0,02 \\
\hline Troponin I (pg/ml) & $0,00-15,60$ & $<10$ \\
\hline
\end{tabular}

an increased hematoma volume in the right occipital lobe with slight compression of the ipsilateral ventricle (Fig. 1). No neurosurgical indication was given, and Mannitol was added to therapy.

During the following days patient's clinical condition improved, with the complete recovery of the state of consciousness, while left-sided lateral hemianopia with color blindness persisted. Subsequent brain CT scans demonstrated the physiological evolution of the hematomas (Fig. 1). Mannitol and Levetiracetam were progressively reduced and then stopped. Blood pressure was stabilized after the optimization of antihypertensive therapy. Also, the general condition improved. Supplemental oxygen was interrupted and after two consecutive nasopharyngeal swabs resulted negative for SARS-CoV-2, patient was transferred to a rehabilitation facility.

\section{Discussion}

Posterior Reversible Encephalopathy Syndrome (PRES) is clinico-radiological disorder related to the rapid development of subcortical vasogenic edema involving mainly parieto-occipital regions with a symmetric distribution [7]. Clinical manifestations of PRES are heterogeneous, may occur in acute or subacute manner and include headache, seizures, visual disturbances and focal neurological deficits. These findings, in association with well-defined risk factors-e.g. renal failure, blood pressure fluctuations, malignancies, immunosuppressive agents, cytotoxic drugs, autoimmune disorders, sepsis, pre-eclampsia or eclampsia-should prompt the diagnosis of PRES [8]. PRES most frequently manifests as bilateral and symmetric vasogenic 
edema within the parietal and occipital lobes, affecting subcortical white matter, although atypical patterns involving the frontal lobes or the cerebellum have been described [9]. Despite clinical features and highly suggestive MRI findings, it is a frequently misdiagnosed clinical condition and epidemiological data are still inconclusive [10]. All age groups may be involved and there is a female predominance even after the exclusion of pregnancy-related PRES [8]. From a clinical standpoint, as the name suggests, it is a reversible condition in $75-90 \%$ of cases, whenever predisposing conditions may be identified and removed [11]. On the other hand, the complete resolution of the neuroradiological findings could not occur in all patients, irrespective of their clinical status. In 10-20\% of cases, permanent neurological sequelae can be present, while death is a rare complication-i.e. 3-6\% - and can be due to intracranial haemorrhage, brain edema causing increased intracranial pressure with brainstem compression or acute hydrocephalus [8]. A high index of suspicion is required even for patients with well-known risk factors-such as patients affected by autoimmune disorders on immunomodulatory treatments-and a prompt diagnosis is mandatory since it may significantly impact the prognosis [7].

Pathophysiological mechanisms are still unclear and other rare neurological diseases, such as reversible cerebral vasoconstriction syndrome (RCVS), may share some clinical features with PRES and occasionally they may be associated [12]. For sure, hypertension and acute hemodynamic stress but also endothelial factors may play a key role in the development of PRES. Cerebrovascular autoregulation is a powerful mechanism providing a constant cerebral blood flow, despite of systemic blood pressure fluctuations, through the release of vasoactive substances [10]. The rapid increase of blood pressure with failure of cerebral autoregulation and subsequent reduction of blood perfusion may induce the leakage of blood-brain barrier (BBB) and then vasogenic edema $[13,14]$. It is more debated the role of vasoconstriction-another cause of reduction of blood perfusion-that is probably a putative mechanism more predominant in RCVS [12]. However, since posterior circulation receives less sympathetic innervation, parietal and occipital lobes are more prone to suffer from hypoperfusion when cerebral vasoconstriction occurs-e.g. in case of the rapid development of systemic high blood pressure [8]. Another factor to consider is endothelial dysfunction related to circulating endogenous or exogenous toxins that could impair BBB in PRES cases in which blood pressure is normal-i.e. about one-third of all patients $[9,15]$. Pial vessels rupture or reperfusion injury, in most severe cases, may lead to cytotoxic edema/infarction or haemorrhagic evolution [16]. The presence of extensive vasogenic edema-seen as T2-weighted or FLAIR hyperintensities typically without restricted diffusion on MRI—or haemorrhage is an adverse prognostic factor and is associated with higher mortality and with a higher modified Rankin Scale score at discharge [17].

The concurrent treatment with anticoagulants or antiplatelets increases the risk of haemorrhagic evolution of PRES, since hypoperfusion makes the brain tissue more fragile. Usually, haemorrhage is a late complication of PRES. In our case, PRES manifested as cortical blindness and encephalopathy followed by bilateral occipital lobe haemorrhages. Against all odds, despite the haemorrhagic PRES and the simultaneous COVID-19-related pneumonia, patient clinical condition improved and she was discharged at a rehabilitation facility. We can assume that the prompt reversal of anticoagulation was a key determinant of neurological prognosis. Up to now, there is no evidence that SARS-CoV-2 infection aggravates the prognosis of patients with PRES. Indeed, to the best of our knowledge, only five cases of COVID-19-related haemorrhagic PRES-including this one-have been published so far (Table 2). Of them, three recovered well despite COVID-19 respiratory impairment, while no information is available for the other two. For three patients the diagnosis was made through MRI and in all cases CT angiography or MR angiography was performed to exclude a major vessel occlusion. The most common clinical presentation was altered mental status (100\% of cases) - the hallmark of encephalopathy-and in our case, it was associated with cortical blindness. No information is available about antithrombotic treatment, even if the use of anticoagulation is a mainstay of COVID-19 therapy [18] and it can be assumed that these patients were on treatment with enoxaparin or similar at the time of symptoms onset.

Acute cerebrovascular diseases affect $1-3 \%$ of hospitalized patients with SARS-CoV-2 infection and up to $6 \%$ of those in ICU [19]. This holds true particularly for adultswith a mean age at time of the event of 63 years oldalthough stroke in the young has anecdotally been reported [5]. According to an observational study on a multinational cohort of COVID-19 patients, there is a significant increase in cryptogenic ischemic stroke (42\%). This may be probably due to embolic mechanisms, related to two main factors: (i) hyperinflammatory state from cytokine storm in the initial phase of respiratory symptoms, (ii) increased prothrombotic state during the late phase of infection [20]. According to another retrospective study on COVID-19 patients, the prevalence of PRES is $1.1 \%$ and in two-third of cases, microhaemorrhages on MRI SWI-sequence could be seen [21].

It is becoming clearer that cytokine storm plays a significant role in the pathogenesis of COVID-19, especially for severely ill patients. The cytokine storm is defined as the inflammatory response induced by COVID-19 leading to the release of a huge number of cytokines and proinflammatory products, including interleukin-1 $\beta$, interleukin-6 (IL-6), TNF, interferon- $\gamma$, and VEGF [22]. Not surprisingly, thromboembolic events seem to be more frequent in critically ill 


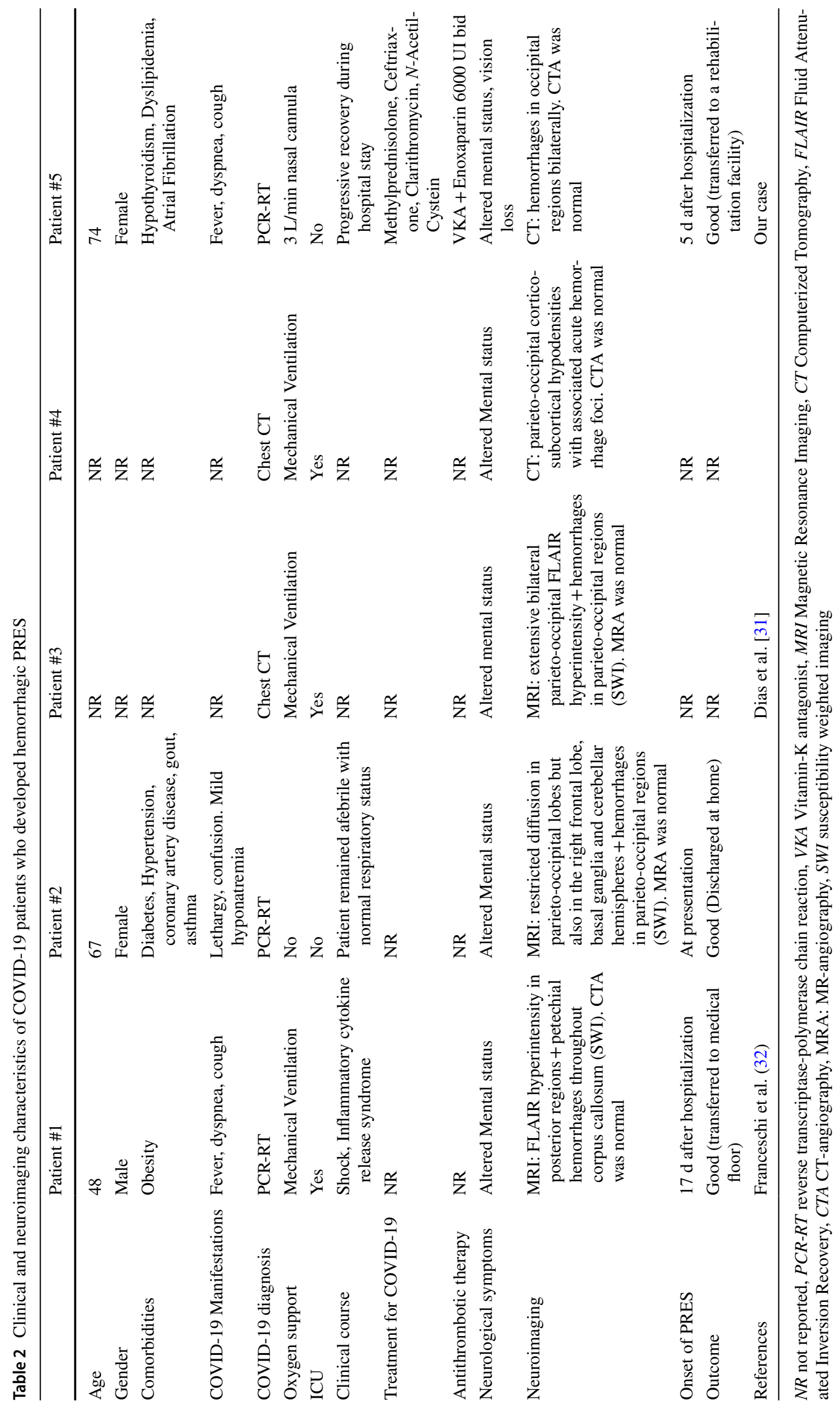


patients with COVID-19-associated cytokine storm [23]. In fact, the exuberant immune response favors the formation of blood clots, thus pulmonary embolism, deep-vein thrombosis, stroke or myocardial infarction are frequent eventsoccurring in almost half of ICU patients with SARS-CoV-2. Cytokine storm however may be crucial in the release of massive amount of vasoconstrictive agents such as thromboxane A2 favoring the onset of PRES (Fig. 2).

Elevation in D-Dimer levels (up to four times the threshold value), slight increase in prothrombin time and platelet count are common hallmarks of dysregulation of the coagulation profile in SARS-CoV-2 infection [24]. In particular, D-dimer levels appear to serve as an indicator of disease progression and severity according to increasing evidence [25]. The pathogenesis of COVID-19 coagulopathy is not yet fully elucidated; however, multiple factors are thought to be implied. Among the molecules released during an inflammatory state, IL-6 induces a wide spectrum of proteins, including fibrinogen and thrombopoietin, and promotes molecular pathways that activate endothelial cell membrane [26]. Thus, thromboembolic events may be also associated with endothelial dysfunction. It has been speculated that the expression of ACE-2 receptor on endothelial cell makes them vulnerable to SARS-CoV-2 invasion. Histopathological studies on tissues of different organs have shown signs of viral invasion, the presence of inflammatory cells, and apoptotic bodies within endothelial cells. These findings have led to the conclusion that the virus could directly cause microvascular dysfunction and ultimately thrombosis [27].

Updated evidence shed a light on the key role of endothelial dysfunction in the pathogenesis of cerebrovascular events. SARS-CoV-2 binds to ACE-2 receptor-a widely distributed receptor on smooth muscle and endothelial cells in the brain-via its Spike protein. Viral invasion, via Spike-protein binding and endocytosis, deplete the ACE-2 receptors. In turn, ACE-2 receptors physiologically bind and cleave Angiotensin II to angiotensin 1-7, that promotes vasodilatation and has anti-inflammatory effects. With ACE-2 depletion, Angiotensin II is free to bind to ACE-1 receptors leading to vasoconstriction, procoagulant and proinflammatory response [28].

In a study of Kaneko et al., endothelial cells obtained from human brains were analyzed by RNA-sequencing. The expression of ACE-2 receptors in brain endothelium were relatively low compared with pulmonary and vasal cells, making clear that the presence of other factors could explain brain vulnerability to COVID-19 invasion. Moreover, SARS-CoV-2 viral S protein binding promotes a unique gene profile expression in cerebral endothelium, that influences directly the immune-mediate response [29].

The alteration of the coagulation profile in COVID-19 includes also haemorrhagic events [30]. Apart from the wellknown use of anticoagulation therapy even in the earliest stage of the disease, a rapid switch from a hypercoagulable

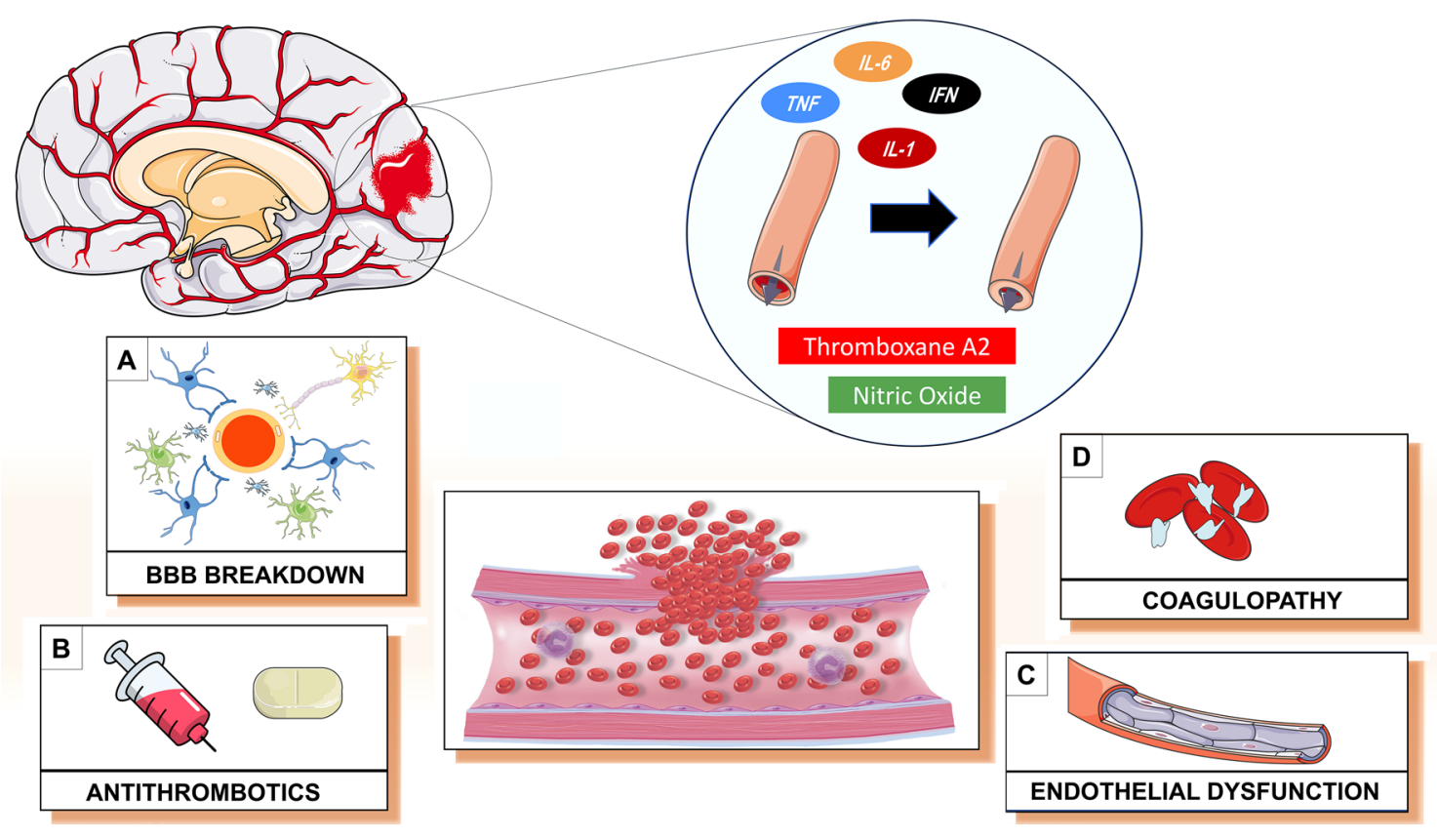

Fig. 2 Different factors contribute to the hemorrhagic evolution of PRES in COVID-19 patients. The reduction in cerebral blood perfusion may be due to the release of vasoactive and proinflammatory molecules in response to COVID-19-related immune activation (round panel). The hypoperfusion determines the breakdown of blood-brain barrier (panel A) that together with antithrombotic treatment (panel B) - a mainstay of SARS-CoV-2 infection therapyendothelial dysfunction (panel $\mathbf{C}$ ) and coagulopathy (panel D)-both related to the immune response-contribute to the development of hemorrhage 
state to bleeding diathesis may occur, as it happens in disseminated intravascular coagulopathy [30]. For these reasons, a prompt interruption/reversal of antithrombotic therapy can be decisive for a good outcome-as occurred in our case. When coagulopathy is too severe, the prognosis is poor [30].

In conclusion, PRES should not be neglected in COVID19 patients who show focal neurological deficits, altered mental status or visual disturbances, because it is a potentially treatable disease when the diagnosis is suspected. Coagulopathy and endothelial dysfunction resulting from the massive release of cytokines during the host immune response may be key factors in the pathogenesis of COVID19-related PRES. Antithrombotic therapy - a mainstay of SARS-CoV-2 treatment-and the leakage of the BBB can subsequently increase the risk of haemorrhagic transformation of the lesioned brain tissue. In this regard-as far as available literature shows- the timely interruption/reversal of antithrombotic therapy may be a key determinant for a good prognosis.

Funding The authors deny any funding source for the present study.

\section{Declarations}

Conflicts of interest Dr. Francesco Motolese reports no disclosures; Dr. Mario Ferrante reports no disclosures; Dr. Mariagrazia Rossi reports no disclosures; Dr. Alessandro Magliozzi reports no disclosures; Dr. Martina Sbarra reports no disclosures; Dr. Francesca Ursini reports no disclosures; Dr. Massimo Marano reports no disclosures; Dr. Fioravante Capone reports no disclosures; Dr. Francesco Travaglino reports no disclosures; Dr. Raffaele Antonelli Incalzi reports no disclosures; Dr. Vincenzo Di Lazzaro reports no disclosures; Dr. Fabio Pilato reports no disclosures.

\section{References}

1. Ellul MA, Benjamin L, Singh B et al (2020) Neurological associations of COVID-19. Lancet Neurol 19:767-783. https://doi.org/ 10.1016/S1474-4422(20)30221-0

2. Dono F, Nucera B, Lanzone J et al (2021) Status epilepticus and COVID-19: a systematic review. Epilepsy Behav 118:107887. https://doi.org/10.1016/j.yebeh.2021.107887

3. Aghagoli G, Gallo Marin B, Katchur NJ et al (2020) Neurological involvement in COVID-19 and potential mechanisms: a review. Neurocrit Care. https://doi.org/10.1007/s12028-020-01049-4

4. Lee M-H, Perl DP, Nair G et al (2021) Microvascular injury in the brains of patients with COVID-19. N Engl J Med 384:481-483. https://doi.org/10.1056/NEJMc2033369

5. Fifi JT, Mocco J (2020) COVID-19 related stroke in young individuals. Lancet Neurol 19:713-715. https://doi.org/10.1016/ S1474-4422(20)30272-6

6. Mishra S, Choueka M, Wang Q et al (2021) Intracranial hemorrhage in COVID-19 patients. J Stroke Cerebrovasc Dis 30:105603. https://doi.org/10.1016/j.jstrokecerebrovasdis.2021.105603

7. Pilato F, Calandrelli R, Distefano M et al (2019) Acute radiological pattern and outcome in posterior reversible encephalopathy syndrome patients. Clin Neurol Neurosurg 185:105459. https:// doi.org/10.1016/j.clineuro.2019.105459

8. Fugate JE, Rabinstein AA (2015) Posterior Reversible Encephalopathy Syndrome: clinical and radiological manifestations, pathophysiology, and outstanding questions. Lancet Neurol 14:914-925. https://doi.org/10.1016/S1474-4422(15)00111-8

9. Bartynski WS, Boardman JF (2007) Distinct imaging patterns and lesion distribution in Posterior Reversible Encephalopathy Syndrome. Am J Neuroradiol 28:1320-1327. https://doi.org/10. 3174/ajnr.A0549

10. Fischer M, Schmutzhard E (2017) Posterior Reversible Encephalopathy Syndrome. J Neurol 264:1608-1616. https://doi.org/10. 1007/s00415-016-8377-8

11. Frick D, Huecker M, Shoff H (2017) Posterior Reversible Encephalopathy Syndrome presenting as stroke mimic. Clin Pract Cases Emerg Med 1:171-174. https://doi.org/10.5811/cpcem.2017.1. 30607

12. Pilato F, Distefano M, Calandrelli R (2020) Posterior Reversible Encephalopathy Syndrome and reversible cerebral vasoconstriction syndrome: clinical and radiological considerations. Front Neurol. https://doi.org/10.3389/fneur.2020.00034

13. Strandgaard S, Paulson OB (1984) Cerebral autoregulation. Stroke 15:413-416. https://doi.org/10.1161/01.STR.15.3.413

14. Li Y, Gor D, Walicki D et al (2012) Spectrum and potential pathogenesis of reversible posterior leukoencephalopathy syndrome. J Stroke Cerebrovasc Dis 21:873-882. https://doi.org/10.1016/j. jstrokecerebrovasdis.2011.05.010

15. Gewirtz AN, Gao V, Parauda SC, Robbins MS (2021) Posterior Reversible Encephalopathy Syndrome. Curr Pain Headache Rep 25:19. https://doi.org/10.1007/s11916-020-00932-1

16. Hefzy HM, Bartynski WS, Boardman JF, Lacomis D (2009) Hemorrhage in Posterior Reversible Encephalopathy Syndrome: imaging and clinical features. Am J Neuroradiol 30:1371-1379. https:// doi.org/10.3174/ajnr.A1588

17. Schweitzer AD, Parikh NS, Askin G et al (2017) Imaging characteristics associated with clinical outcomes in Posterior Reversible Encephalopathy Syndrome. Neuroradiology 59:379-386. https:// doi.org/10.1007/s00234-017-1815-1

18. Akima S, McLintock C, Hunt BJ (2020) RE: ISTH interim guidance to recognition and management of coagulopathy in COVID19. J Thromb Haemost 18:2057-2058. https://doi.org/10.1111/jth. 14853

19. Iadecola C, Anrather J, Kamel H (2020) Effects of COVID-19 on the nervous system. Cell 183:16-27.e1. https://doi.org/10.1016/j. cell.2020.08.028

20. Yaghi S, Ishida K, Torres J et al (2020) SARS-CoV-2 and stroke in a New York healthcare system. Stroke 51:2002-2011. https:// doi.org/10.1161/STROKEAHA.120.030335

21. Lin E, Lantos JE, Strauss SB et al (2020) Brain imaging of patients with COVID-19: findings at an academic institution during the height of the outbreak in New York city. Am J Neuroradiol 41:2001-2008. https://doi.org/10.3174/ajnr.A6793

22. Fajgenbaum DC, June CH (2020) Cytokine storm. N Engl J Med 383:2255-2273. https://doi.org/10.1056/NEJMra2026131

23. Klok FA, Kruip MJHA, van der Meer NJM et al (2020) Confirmation of the high cumulative incidence of thrombotic complications in critically ill ICU patients with COVID-19: an updated analysis. Thromb Res 191:148-150. https://doi.org/10.1016/j.thromres. 2020.04.041

24. Zhang L, Yan X, Fan Q et al (2020) D-dimer levels on admission to predict in-hospital mortality in patients with COVID-19. J Thromb Haemost 18:1324-1329. https://doi.org/10.1111/jth. 14859

25. Becker RC (2020) COVID-19 update: COVID-19-associated coagulopathy. J Thromb Thrombolysis 50:54-67. https://doi.org/ 10.1007/s11239-020-02134-3 
26. Shi W, Lv J, Lin L (2020) Coagulopathy in COVID-19: focus on vascular thrombotic events. J Mol Cell Cardiol 146:32-40. https:// doi.org/10.1016/j.yjmcc.2020.07.003

27. Varga Z, Flammer AJ, Steiger P et al (2020) Endothelial cell infection and endotheliitis in COVID-19. Lancet 395:1417-1418. https://doi.org/10.1016/S0140-6736(20)30937-5

28. Gavriilaki E, Anyfanti P, Gavriilaki M et al (2020) Endothelial dysfunction in COVID-19: lessons learned from coronaviruses. Curr Hypertens Rep 22:63. https://doi.org/10.1007/ s11906-020-01078-6

29. Kaneko N, Satta S, Komuro Y et al (2021) Flow-mediated susceptibility and molecular response of cerebral endothelia to SARSCoV-2 infection. Stroke 52:260-270. https://doi.org/10.1161/ STROKEAHA.120.032764
30. Iba T, Levy JH, Levi M, Thachil J (2020) Coagulopathy in COVID-19. J Thromb Haemost 18:2103-2109. https://doi.org/ 10.1111/jth. 14975

31. Dias DA, de Brito LA, Neves LO, Paiva RGS, Barbosa Júnior OA, Tavares-Júnior JWL (2020) Hemorrhagic PRES: an unusual neurologic manifestation in two COVID-19 patients. Arq Neuropsiquiatr 78(11):739-740. https://doi.org/10.1590/0004-282X2 0200184

32. Franceschi AM, Ahmed O, Giliberto L, Castillo M (2020) Hemorrhagic posterior reversible encephalopathy syndrome as a manifestation of COVID-19 infection. AJNR Am J Neuroradiol 41(7):1173-1176. https://doi.org/10.3174/ajnr.A6595 